\title{
Lymphocytic interstitial pneumonia in a patient with lymphocytic colitis
}

\author{
Cezary Chojnacki ${ }^{1}$, Alina Knopik-Dąbrowicz ${ }^{2}$, Dorota Mania ${ }^{3}$, Jan Chojnacki ${ }^{1}$ \\ ${ }^{1}$ Department of Clinical Nutrition and Gastroenterological Diagnostics, Medical University of Lodz, Lodz, Poland \\ 2Department of Pathomorphology and Cytology, Medical University of Lodz, Lodz, Poland \\ ${ }^{3}$ Department of Imaging Diagnostics, Military Medical Hospital, Lodz, Poland
}

Gastroenterology Rev 2022; 17 (3): 250-252

DOI: https://doi.org/10.5114/pg.2022.112778

\begin{abstract}
Address for correspondence: Cezary Chojnacki PhD, Department of Clinical Nutrition and Gastroenterological Diagnostics, Medical University of Lodz, Lodz, Poland, phone: +48 506566 820, e-mail: cezary.chojnacki@umed.lodz.pl
\end{abstract}

The course of lymphocytic colitis (LC) is usually mild with periods of exacerbation and remission. Chronic non-bloody diarrhoea [1] dominates. In recent years significant findings with respect to the association of the gut intraepithelial lymphocytes (IELS) and various diseases have been observed [2]. We report the cause of LC with coexistence of severe lymphocytic interstitial pneumonia (LIP).

A 61-year-old female with a long history of the disease was admitted to the Department of Gastroenterology due to chronic diarrhoea and shortness of breath during exercise. At the age of 20 years, she started to complain of abdominal pain and recurrent diarrhoea. At that time, the results of diagnostic tests indicated irritable bowel syndrome. Allergy, food intolerances, and bacterial infections were excluded. Laboratory test results were normal. The macroscopic picture of the colon was also unchanged, histological examination of the colonic mucosa revealed an increase in the number of intraepithelial lymphocytes without structural changes, and nonspecific colitis was diagnosed. At that time, a $2.0 \mathrm{~g}$ daily dose of mesalazine was added, and she took it for 24 months. In the following years, abdominal pain and loose stools recurred sporadically and the patient did not take anti-inflammatory drugs. From 55 years of age, her health condition systematically deteriorated. Diarrhoea and abdominal pain worsened. In addition, the patient has been diagnosed with other immune-inflammatory diseases, i.e. thyroiditis, myositis, arthritis, and axonal polyneuropathy. In particular, exertional dyspnoea increased, and she was admitted to the Department of Pulmonology.

Gasometry showed partial pressure of oxygen $\left(\mathrm{PO}_{2}\right)$

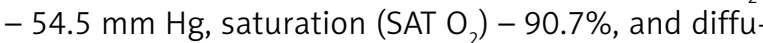
sion abnormalities for CO (DLCO) $-42 \%$ of normal, and spirometry revealed reduced $\mathrm{FEV}_{1}$ to $68 \%$ of normal. Next, a high-resolution computed tomography (HRCT) image of the lung was performed, and generalized interstitial changes were observed, with predominance of ground-glass opacification with inter-lobular septal thickening and reticulation, especially in the lower lung zone. Furthermore, slight apical fibrous changes and emphysematous bullae at the base of the lungs were seen, wirth normal mediastinal structure without signs of adenopathy (Figure1 A).

The picture suggested viral pneumonia. For this reason, real time polymerase chain reaction (RT-PCR) test for viral infection of SARS-CoV-2 was performed, and COVID-19 disease was excluded. Subsequently, bronchoscopy and lung tissue biopsy were performed. The image of airways was normal, whereas $71.7 \%$ of lymphocytes (norm $<15$ ) and $1.0 \%$ of monocytes (norm $<0.5)$ were found in bronchoalveolar lavage (BAL). Histopathological examination: The microscopic image corresponds to diffuse interstitial inflammatory changes with infiltration from CD3 (+), CD20 (-) T cells, and CD138 (+) plasma cells. Conclusions: Lymphoid interstitial pneumonia (LIP), which may be associated with systemic or immune-mediated diseases. No specific antibodies were detected against the following: RNP/Sm, Sm, SS-a native, RO-52, SS-B, Sci-70, PM-Scl, Jo-1, Centromere B, PCNA, for DNA, nucleosomes, histones, ribosomal $P$ protein, $A M A-M 2$, and DFS70. Blood concentration of albumins and globulins and their percentage composition, including the IgG1 - IgG4 fraction, did not deviate from normal levels. The level of C-reactive protein (CRP) ranged from 6.1 to $8.0 \mathrm{mg} / \mathrm{l}$. The results of routine laboratory tests were normal except for a slight increase in alanine aminotransferase activity within the range 49-66 IU/I. In recent years ab- 

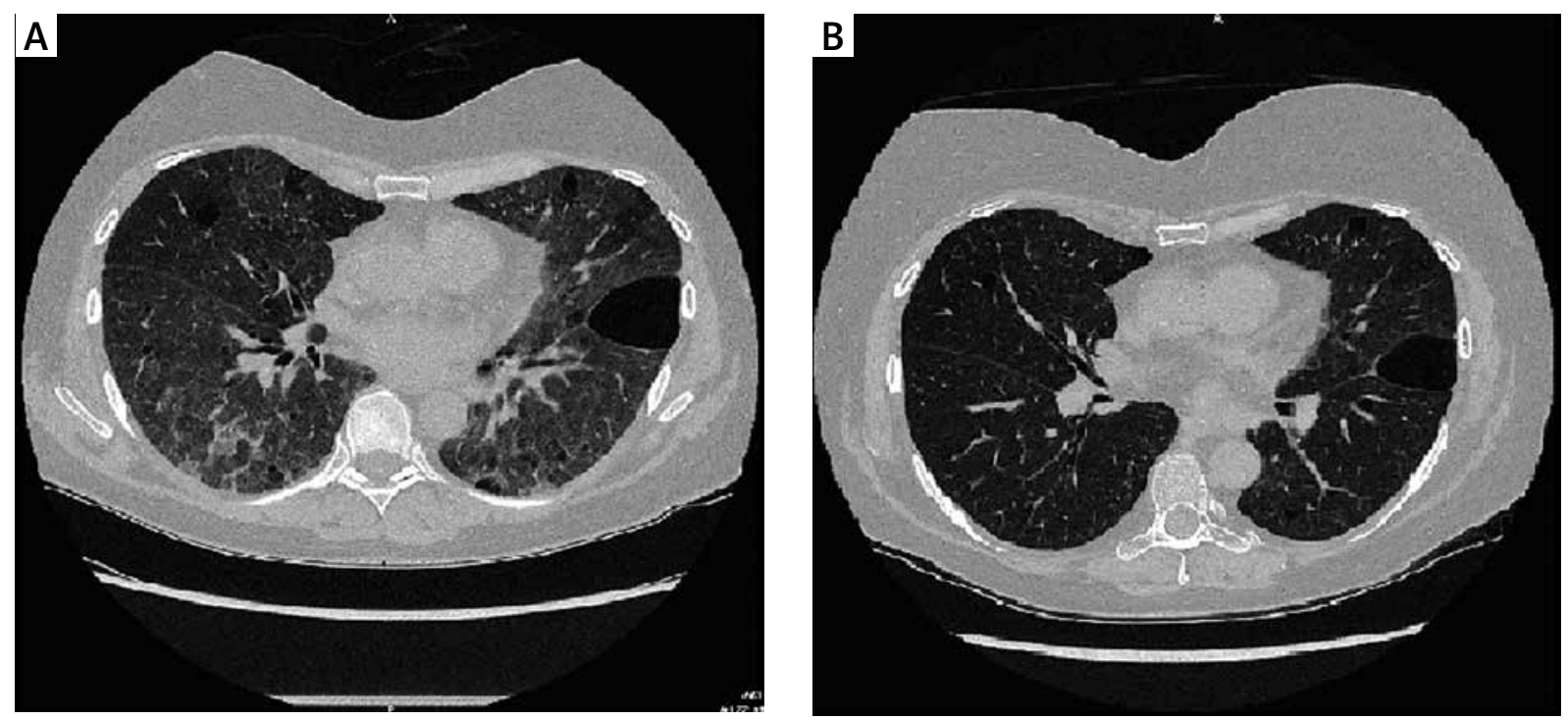

Figure 1. A - Image of lungs: generalized interstitial changes, with predominance of ground-glass opacification, with inter-lobar septal thickening and reticulation, and with emphysematous bullae. B - The image of lungs after 3 months of immune-therapy; revealed resolution of ground-glass opacification and reticulation as well as less pronounced interlobular thickening

dominal pain and diarrhoea developed and the patient has been diagnosed in Department of Gastroenterology, and a clinical examination was performed. Again, allergy, food intolerance, coeliac disease, and dysbiosis were excluded. In the microscopic picture of colonic mucosa, the number of IELs was increased to an average of 40/100 enterocytes.

In addition, lymphocyte and plasma cell infiltration was seen in the glandular zone. After analysis of the above results, the following treatment was recommended: gluten-free and low-tryptophan diet, prednisone at a daily dose of 45-15 mg (step down), followed by deflazacort (15 mg to $9 \mathrm{mg}$ ) and azathioprine (150$100 \mathrm{mg}$ ). Additionally, levothyroxine (75 mg), sodium butyrate $(500 \mathrm{mg})$, and a combination of vitamins $-B_{1}$, $B_{6}$, and $B_{12}-$ were administered. Currently, mesalazine is not recommended in this disease. After 3 months, the patient's condition improved. In particular, myalgia and arthralgia, abdominal pain, and diarrhoea resolved. Exertional dyspnoea decreased, $\mathrm{FEV}_{1}$ increased from $59 \%$ to $68 \%$, and DLCO from $42 \%$ to $63 \%$ of normal. HRCT revealed resolution of ground-glass opacification and reticulation as well as less pronounced interlobular septal thickening (Figure $1 \mathrm{~B}$ ). The number of IELS in sigmoid mucosa decreased to $23 / 100 \mathrm{EC}$, and faecal calprotectin decreased from $239 \mu \mathrm{g} / \mathrm{g}$ to $4.86 \mu \mathrm{g} / \mathrm{g}$. The patient continues to take the above medications and remains under constant medical supervision.

In our patients, LIP is a serious life-threatening disease. This disease is rarely spontaneous, but it accompanies other immune and inflammatory diseases.
COVID-19 disease, HIV, HBV, HCV, and EBV infections as well as Sjögren's disease and rheumatoid arthritis were excluded in the patient. Systemic diseases, i.e. myeloma, lymphoma, and lymphocytic leukaemia, were also excluded. Concurrent changes in the lungs, thyroid, and nervous system occur in temporal association with LC. However, it is not certain whether these changes are causal, but it cannot be ruled out. Gastrointestinal mucosa is particularly exposed to adverse external factors, including food, and bacterial and viral antigens. Therefore, the body develops its own defence system, which includes lymph nodes, Peyer's patches, isolated lymph follicles and diffused $T$ and B lymphocytes, macrophages, mast cells, plasma cells, and granulocytes. In LC there comes to the increase in the number of intraepithelial lymphocytes, predominantly CD8, which are involved in activation of innate and acquired immunity. IL cells secrete many cytokines, the main function of which is a regulatory role [3]. Moreover, all subpopulations of intraepithelial lymphocytes can migrate beyond the intestinal wall. Constant lymphocyte recirculation between the circulatory system and lymphatic tissues is necessary for proper functioning of the immune system [4]. The effectiveness of immune response, whether primary or secondary, depends among others on the ability of lymphocytes to reach the focus of infection. The lymphocyte stimulated by the right stimulus changes its volume and increases the production of lymphokines, which are necessary for the development of further stages of the immune response, i.e. proliferation and differentiation. Each immune reaction is a chain of de- 
pendencies, and its links are, among others, activation, adhesion, and migration. It can be assumed that local immune processes are triggered as a result of active lymphocyte migration from the gastrointestinal tract to the lungs, thyroid, muscles, or nervous system. It is believed that despite the space between individual elements of the lymphoid tissue, they form one common immune system (mucosa-associated lymphoid tissue - MALT) [4]. This concept assumes that lymphocytes derived from one part of this system change location, colonize its other surfaces, which leads to activation of $B$ cells in various parts of MALT. In this sense, LC can be regarded as a generalized immune and inflammatory disease. Depending on the degree and stage of its advancement, there are various clinical manifestations requiring complex treatment, but usually budesonide is recommended [5]. When other autoimmune diseases co-exist, as in the case of our patient, it was necessary to administer systemic glucocorticosteroids and immunosuppressive drugs. A significant improvement in the lungs, as well in the colon, after 3 months of the treatment confirms the immunological nature of the described organ changes.

In conclusion, the presented case confirms that LC is a chronic autoimmune and inflammatory disease, not devoid of various complications, which should be intensively treated and monitored.

\section{Conflict of interest}

The authors declare no conflict of interest.

\section{References}

1. Park T, Cave D, Marshall C. Microscopic colitis: a review of etiology, treatment and refractory disease. World J Gastroenterol 2015; 21: 8804-10.

2. Pisani LF, Tontini GE, Vecchi M, et al. Microscopic colitis: what do we know about pathogenesis? Inflamm Dis 2016; 22: 450-8.

3. Sonnenberg A, Turner KO, Genta RM. Associations of microscopic colitis with other lymphocytic disorders of the gastrointestinal tract. Clib Gastroenterol Hepatol 2018; 16: 1762-7.

4. Ma Y, Yang X, Chatterjee V, et al. The gut-lung axis in systemic inflammation. Role of mesenteric lymph as a conduit. Am J Respir Cell Mol Biol 2021; 64: 19-28.

5. Miehlke S. European guidelines on microscopic colitis: United European Gastroenterology and European Microscopic Colitis Group statements and recommendations. United European Gastroenterol J 2021; 9:3-37.

Received: 21.10 .2020

Accepted: 5.11.2021 